\title{
Perfil dos usuários de varfarina atendidos em uma farmácia pública de um município
}

\section{do interior da Bahia}

\author{
Profile of warfarin users served in a public pharmacy in amunicipality in the interior of Bahia \\ Perfil de usuarios warfarin atendidos en farmacia pública en unmunicipio del interior de Bahia
}

Recebido: 2611/2021 | Revisado: 30/11/2021 | Aceito: 01/12/2021 | Publicado: 04/12/2021

\author{
Daianne Arruda Luz \\ ORCID: https://orcid.org/0000-0002-2734-2612 \\ Faculdade Independente do Nordeste, Brasil \\ E-mail: daianne.arruda13@gmail.com \\ Danielle Portugal Vieira \\ ORCID: https://orcid.org/0000-0002-9511-534X \\ Faculdade Independente do Nordeste, Brasil \\ E-mail: dany_portugal@outlook.com.br \\ Nicolly Luz Pita \\ ORCID: https://orcid.org/0000-0002-4944-832X \\ Faculdade Independente do Nordeste, Brasil \\ E-mail: nicollyluz@gmail.com \\ Rodrigo Santos Damascena \\ ORCID: https://orcid.org/0000-0002-0527-6183 \\ Faculdade Independente do Nordeste, Brasil \\ E-mail: rodrigodamascena@ fainor.com
}

\begin{abstract}
Resumo
Os anticoagulantes são substâncias que impedem a formação de coágulos no sangue, sendo muito utilizados em problemas cardiovasculares. Um dos anticoagulantes orais mais utilizados nos últimos 50 anos é a Varfarina, que tem sua eficácia comprovada na prevenção de algumas doenças, como infarto agudo do miocárdio, tromboembolismo venoso, embolia sistêmica, fibrilação auricular, dentre outras. Apesar de sua eficácia, este medicamento apresenta uma margem terapêutica estreita, onde sua dose terapêutica é muito próxima da letal, podendo assim, ocasionar efeitos tóxicos aos usuários. Deste modo, este estudo tem como objetivo descrever o perfil dos usuários da varfarina atendidos na farmácia pública de uma Unidade Básica de Saúde, em Vitória da Conquista (município do interior da Bahia). Trata- se de um estudo quantitativo e de caráter transversal com delineamento descritivo, realizado em 10 participantes. Foramaplicados questionários para os usuários da varfarina será feita a análise desses dados coletados, seguindo todas as questões éticas do estudo. Os resultados desta pesquisa desvelam um perfil de mais homens que mulheres, onde os motivos do uso da varfarina entre eles são bem comuns, endo $60 \%$ por AVC, $20 \%$ dos participantes entrevistados usamo medicamento há mais de 12 meses, e também faz uso de outros medicamentos simultaneamente, que é um fator importantíssimo a ser acompanhado para que não ocorra nenhuma interação medicamentosa e diminua também os risco das reações adversas. Conclui-se que o medicamento em questão é de suma importância para todos que utilizam, e sendo assim, este estudo vem nos acrescentar a importância do acompanhamento na terapia anticoagulante, devido os riscos que a mesma pode gerar ao paciente, nenhum resultado obtido foi absoluto ou pode ser considerado $100 \%$ assertivo, por isso se faz necessária mais pesquisas relacionadas ao tema em maiores populações e amostras, através de coletas de dados mais abrangentes nas unidades básicas de sáude em geral, para a realização de artigos ou trabalhos futuros.
\end{abstract}

Palavras-chave: Anticoagulante; Varfarina; Farmacoterapia; Coagulação.

\begin{abstract}
Anticoagulants are substances that prevent the formation of clots in the blood, being widely used in cardiovascular problems. One of the most used oral anticoagulants in the last 50 years is Warfarin, which has been proven to be effective in preventing some diseases, such as acute myocardial infarction, venous thromboembolism, systemic embolism, atrial fibrillation, among others. Despite its effectiveness, this drug has a narrow therapeutic margin, where its therapeutic dose is very close to lethal, and thus, it can cause toxic effects to users. Thus, this study aims to describethe profile of warfarin users treated at the public pharmacy of a Basic Health Unit, in Vitória da Conquista (a municipality in the interior of Bahia). This is a quantitative and cross-sectional study with a descriptive design, carried out with 10 participants. Questionnaires were applied to warfarin users, the data collected will be analyzed, following all the ethical questions of the study. The results of this research reveal a profile of more men than women, where the reasons for the use of warfarin among them are very common, being $60 \%$ for stroke, $20 \%$ of the interviewed participants have used the drug for more than 12 months, and also use it. of other drugs simultaneously, which is a
\end{abstract}


veryimportant factor to be monitored so that no drug interactions occur and also reduce the risk of adverse reactions. It is concluded that the drug in question is of paramount importance for all who use it, and therefore, this study adds to the importance of monitoring the anticoagulant therapy, due to the risks it can generate for the patient, no result obtained was absolute or it can be considered $100 \%$ assertive, which is why more research related to the topic in larger populations and samples is needed, through more comprehensive data collection in basic health units in general, for future articles or work.

Keywords: Anticoagulant; Warfarin; Pharmacotherapy; Coagulation.

\section{Resumen}

Los anticoagulantes son sustancias que evitan la formación de coágulos en la sangre y se utilizan ampliamente en problemas cardiovasculares. Uno de los anticoagulantes orales más utilizados en los últimos 50 años es la warfarina, que ha demostrado su eficacia en la prevención de algunas enfermedades, como infarto agudo de miocardio, tromboembolismo venoso, embolia sistémica, fibrilación auricular, entre otras. A pesar de su eficacia, este fármaco tiene un rango terapéutico estrecho, donde su dosis terapéutica es muy cercana a la letal y, por tanto, puede provocar efectos tóxicos en los usuarios. Así, este estudio tiene como objetivo describir el perfil de los usuarios de warfarina atendidos en la farmacia pública de una Unidad Básica de Salud, en Vitória da Conquista (municipio del interior de Bahía). Se trata de un estudio cuantitativo, transversal con un diseño descriptivo, realizado con 10 participantes. Se aplicaron cuestionarios a los usuarios de warfarina, los datos recolectados serán analizados, siguiendo todas las cuestiones éticas del estudio. Los resultados de esta investigación revelan un perfil de más hombres que mujeres, donde los motivos para el uso de warfarina entre ellos son muy comunes, siendo el $60 \%$ por ictus, el $20 \%$ de los participantesentrevistados utilizó el fármaco durante más de 12 meses, y También utilícelo simultáneamente con otros medicamentos, lo cual es un factor muy importante a vigilar para que no se produzcan interacciones medicamentosas ytambién se reduzca el riesgo de reacciones adversas. Se concluye que el fármaco en cuestión es de suma importancia para todas las personas que lo utilizan, por lo que este estudio se suma a la importancia del seguimiento de la terapia anticoagulante, debido a los riesgos que puede generar para el paciente, ningún resultado obtenido fue absoluto o se puede considerar $100 \%$ asertivo, por lo que se necesita más investigación relacionada con el tema en poblaciones y muestras más amplias, a través de una recolección de datos más integral en las unidades básicas de salud en general, para la realización de futuros artículos u obras.

Palabras clave: Anticoagulante; Warfarina; Farmacoterapia; Coagulación.

\section{Introdução}

A coagulação sanguínea é um processo onde o sangue passa do seu estado líquido para um estado onde o mesmo se transforma em uma massa sólida composta pela fibrina, e caso esse processo ocorra dentro de um vaso sanguíneo o mesmo é denominado trombose. Por esse motivo, pessoas que possuem problemas relacionados à coagulação sanguíneapassam a fazer o uso de anticoagulantes orais (Borlina et al., 2010).

Os anticoagulantes são responsáveis por prolongar o tempo de coagulação do sangue, sendo utilizados para muitos problemas cardiovasculares. Dentre eles podem ser destacados os problemas cardíacos, reumáticos, o infarto agudo do miocárdio, a embolia pulmonar, doença vascular cerebral, doença periférica e trombose venosa. Sendo assim, essa classe de medicamentos vai agir inibindo a ação ou formação de um ou mais fatores de coagulação (Riese; Konarzewski; 2017).

Um dos fatores de risco para doenças tromboembólicas é o envelhecimento progressivo da população. Estudos mostraram que as terapias com anticoagulantes orais trouxeram benefícios em pessoas com problemas cardiovasculares, onde a varfarina é o fármaco de escolha para a profilaxia e tratamento de muitas doenças como: embolia pulmonar, trombose venosa, e fibrilação atrial com embolização (Santana et al., 2015).

A Varfarina é um dos fármacos que vem sendo mais utilizado há cerca de 50 anos na terapia com anticoagulantes, onde foi comprovada sua eficácia na prevenção de algumas patologias, dentre elas podemos citar seu uso na prevenção primária do infarto agudo do miocárdio (IAM), tromboembolismo venoso, embolia sistêmica, fibrilaçãoauricular, entre outros. Descobriuse também a importância de seu uso em pessoas que já sofreram IAM, a fim de prevenir que o mesmo ocorra novamente e também de prevenir o Acidente Vascular Cerebral mais conhecido como AVC e/ou até mesmo da morte (Menezes et al., 2015).

Este anticoagulante oral apresenta uma margem terapêutica estreita, o que significa que sua dose terapêutica é muito 
próxima da dose letal, podendo causar efeitos tóxicos aos indivíduos que fazem o uso do mesmo. Sendo sua dose inicial para tratamento $10 \mathrm{mg} /$ dia em adultos durante um período de 2 dias e a dose de manutenção entre $3 \mathrm{mg}$ a $9 \mathrm{mg}$ levando-se em conta o tempo de protrombina, que deve ser monitorado através do INR e a partir de aí serem feitos os devidos ajustes de dose (Riese \& Konarzewski, 2017).

Além de pertencer a uma classe de anticoagulantes mais usados hoje em dia, deve-se ter muita atenção, pois o tratamento com o mesmo apresenta um elevado número de potencial de interação com medicamentos, está na relação dos medicamentos potencialmente perigosos e ainda está entre os 10 fármacos que podem causar reações adversas com medicamentos. Além disso, ainda foi retratado pela literatura o surgimento de interações da varfarina com álcool, cigarro,com fitoterápicos, chás e até mesmo com a dieta alimentar (Santana et al., 2015).

Sendo assim, deve se destacar a importância do acompanhamento farmacêutico para pessoas que fazem terapia com anticoagulantes orais, em especial a varfarina e sobre o uso do mesmo com muita cautela. É necessário levar sempreem conta a orientação do médico e também do farmacêutico, a fim de promover o uso racional do medicamento, e garantindo que o paciente esteja ciente de todos os riscos que a utilização inadequada da varfarina pode causar. Deve-seassegurar que ele esteja recebendo as devidas orientações sobre o fármaco que está utilizando, garantindo que o pacienteesteja recebendo a medicação adequada, na dose correta, baseada em seus requisitos individuais, no tempo correto, minimizando todos os riscos e assim interferindo positivamente na adesão ao tratamento (Menezes et al., 2015). Anteposto, esta pesquisa apresenta como objetivo descrever o perfil dos usuários de Varfarina atendidos em uma farmáciapública de um município do interior da Bahia.

\section{Metodologia}

Trata-se de um estudo quantitativo e de caráter transversal com delineamento descritivo. Os estudos transversais são instrumentos muito utilizados para descrever a característica de uma população, identificar grupos de risco, e para o planejamento em saúde e ação. Pode fornecer dados importantíssimos para o avanço do conhecimento científico, se utilizando como é indicado (Bastos \& Duquia, 2007).

Os estudos descritivos vão descrever as variáveis em estudo que são as características da amostra. É imprescindível para o conhecimento na coleta de resultados de uma instituição estabelecida, no estudo de doenças raras, e também na formação de possibilidades a serem testadas por outros modelos de pesquisa clínica. No estudo descritivo não se tem um grupo de comparação, sendo sua principal característica, onde seu objetivo é descrever um fato médico (Soares et al., 2011).

Este estudo foi realizado em uma Farmácia da Família de um município do interior da Bahia, que se situa no semiárido nordestino. Sua população em 2010 no último censo foi de 306.866 pessoas e em 2020 a população estimada era de 341.128 pessoas (Ibge, 2020).

Foram participantes do estudo pacientes que fazem o uso da terapia anticoagulante oral com varfarina. A amostra foi composta por 10 usuários de varfarina atendidos na farmácia da UBS, onde os usuários menores de 18 anos ou pessoas legalmente incapazes foram excluídos desta pesquisa.

A coleta de dados foi realizada nas farmácias das Unidades Básicas de Saúde. Essa coleta foi realizada a partir dos dados sociodemográficos como: sexo, idade, estado civil, renda, escolaridade, e também através de dados específicos, sendo eles: motivo para utilização da varfarina, dose utilizada, tempo que se usa, se há presença de alguma comorbidadeou não, queixas em relação ao medicamento, origem da prescrição ou se há alguma reação adversa. Os dados coletados foram organizados através do pacote estatístico Statistical Package for the Social Sciences (SPSS) versão 22.0. Sendo assim, realizou-se também uma análise descritiva em forma de frequência absoluta e relativa das variáveis categóricas edesvio padrão e média para as variáveis contínuas estatísticos qui quadrado e de regressão logística para análises bivariadas. 
A pesquisa atendeu aos preceitos éticos, sendo submetida para apreciação pelo Comitê de Ética da Faculdade Independente do Nordeste - CEP/FAINOR, de acordo com as normas dispostas na resolução 466/2012 e 510/2010 do Conselho Nacional de Saúde (CN), e iniciada somente após aprovação sob parecer n. 4.930.579.

\section{Resultados e Discussão}

A pesquisa consistia em 16 perguntas de caráter qualitativo e quantitativo acerca do perfil dos participantes do estudo, sua motivação em utilizar os medicamentos, idade, sexo, posologia, frequência de utilização dos medicamentos, controle de acompanhamento médico, se possuíam comorbidade, qual possuíam, se sentiram reações adversas com os medicamentos, se havia prática de atividade física e se a alimentação era saudável entre os pesquisados como é demonstrado na tabela 1 à baixo:

Tabela 1 - Dados levantados através da pesquisa.

\begin{tabular}{|c|c|}
\hline \multicolumn{2}{|c|}{ Sexo biológico do paciente } \\
\hline Masculino & $40 \%$ (4 pessoas) \\
\hline Feminino & $60 \%$ (6 pessoas) \\
\hline \multicolumn{2}{|c|}{ Motivo do uso da varfarina } \\
\hline Acidente Vascular Cerebral & $60 \%$ (6 pessoas) \\
\hline Problema na Válvula & $20 \%$ (2 pessoas) \\
\hline Trombose Coronária & $10 \%$ (1 pessoa) \\
\hline Insuficiência Cardíaca & $10 \%$ (1 pessoa) \\
\hline
\end{tabular}

\section{Tempo de uso dos medicamentos}

\begin{tabular}{|c|c|}
\hline 120 meses & $10 \%$ (1 pessoa) \\
\hline 72 meses & $10 \%$ (1 pessoa) \\
\hline 60 meses & $10 \%$ (1 pessoa) \\
\hline 48 meses & $10 \%$ (1 pessoa) \\
\hline 36 meses & $10 \%$ (1 pessoa) \\
\hline 12 meses & $20 \%$ (2 pessoas) \\
\hline 8 meses & $10 \%$ (1 pessoa) \\
\hline 6 meses & $10 \%$ (1 pessoa) \\
\hline 2 meses & $10 \%$ (1 pessoa) \\
\hline \multicolumn{2}{|c|}{$\begin{array}{l}\text { Quantidade de medicamentos } \\
\text { tomados }\end{array}$} \\
\hline 2 medicamentos & $10 \%$ (1 pessoa) \\
\hline 3 medicamentos & $20 \%$ (2 pessoas) \\
\hline 4 medicamentos & $50 \%$ (5 pessoas) \\
\hline 5 medicamentos & $10 \%$ (1 pessoa) \\
\hline 6 medicamentos & $10 \%$ (1 pessoa) \\
\hline
\end{tabular}




\begin{tabular}{|c|c|}
\hline \multicolumn{2}{|c|}{ Medicamentos utilizados } \\
\hline Enalapril & $40 \%$ (4 pessoas) \\
\hline Varfarina & $100 \%$ (10 pessoas) \\
\hline Carvediol & $50 \%$ (5 pessoas) \\
\hline Digoxina & $10 \%$ (1 pessoa) \\
\hline Aldactone & $50 \%$ (5 pessoas) \\
\hline Sinvastatina & $30 \%$ (3 pessoas) \\
\hline Losartana & $30 \%$ (3 pessoas) \\
\hline Metformina & $10 \%$ (1 pessoa) \\
\hline Anlodipino & $10 \%$ (1 pessoa) \\
\hline Benzentacil & $10 \%$ (1 pessoa) \\
\hline \multicolumn{2}{|c|}{ Periodicidade das consultas médicas } \\
\hline $\begin{array}{l}\text { A } 1 \text { mês atrás quando iniciou e voltou para a revisão } \\
\text { no mês seguinte }\end{array}$ & $70 \%$ (7 pessoas) \\
\hline $\begin{array}{c}\text { A } 5 \text { meses atrás quando iniciou o tratamento e voltou } \\
\text { para a revisão no mês seguinte }\end{array}$ & $10 \%$ (1 pessoa) \\
\hline A 8 meses atrás quando começou a fazer o tratamento & $10 \%$ (1 pessoa) \\
\hline Vai 2 vezes ao ano & $10 \%$ (1 pessoa) \\
\hline
\end{tabular}

Fonte: Autores (2021).

A partir da coleta sociodemográfica, foi possível entender que, dos 10 participantes da pesquisa $60 \%$ eram do sexo masculino e $40 \%$ do sexo feminino, com variação de idade entre 46 e 77 anos, com média de 58,3 anos, e desvio padrão de idade de $12,19 \%$.

Com relação ao motivo pelo qual os pacientes utilizavam a varfina, $60 \%$ respondeu que utilizava a mesma por Acidentes Vasculares Cerebrais (AVC's) pregressos, alguns relatando também possuírem arritmia e válvulas de metal no coração, $20 \%$ disseram que fazia uso por problemas valvulares, informando já terem feito cirurgia, 10\% relatou que possuíam insuficiências cardíacas e $10 \%$ trombose coronária.

Segundo Colet et al (2016), no Brasil, não existem muitos dados, sobre a dispensação da varfarina, onde o predomínio dos casos de fibrilação atrial entre a população chega a ser cerca de 1\%, e já em 1.000 habitantes/ano, 0,6 casos representam a ocorrência de trombose venosa. Deste modo, este medicamento é utilizado para essas doenças como tratamento prioritário. Colet et al (2016), também destaca que pode ocorrer um aumento do uso da varfarina, levando-seem conta o fato de que este medicamento geralmente é destinado à doenças que vem a acometer em sua maior parte os idosos, e acreditando também no aumento da expectativa de vida.

Dalpiaz et al, (2017), retrata que os motivos recorrentes do uso da varfarina são, a prevenção do tromboembolismo venoso, uso em pacientes com infarto agudo do miocárdio, fibrilação atrial crônica, válvulas cardíacas, é utilizado também durante cirurgias, além disso, evita o desenvolimento e o resurgimento, de trombose aguda de veias profundas, ou embolia pulmonar.

Quando questionados em relação a dose que utilizavam, 100\% dos indivíduos relataram utilizar 5 mg e já relacionado ao tempo de uso, $10 \%$ respondeu fazer uso a 120 meses, $10 \%$ a 72 meses, $10 \%$ a 60 meses, $10 \%$ a 48 meses, $10 \%$ a 36 meses, $20 \%$ a 12 meses, $10 \%$ a 8 meses e $10 \%$ a 6 meses e por fim também $10 \%$ a apenas 2 meses.

Relacionada a quantidade de medicamentos simultaneamente utilizados, 50\% relataram utilizar 4 medicamentos, $20 \%$ 
faziam uso de 3 medicamentos, $20 \%$ utilizava 6 medicamentos simultâneos e $10 \%$ utilizava 5 medicamentos ao mesmo tempo, um fato que deve-se ter bastante cautela, para que não ocorra interações medicamentosas .

Barbosa et al, (2018) destaca que o uso racional dos medicamentos, é de suma importância, em conjunto com a compreensão dos usuários sobre a terapia anticoagulante, juntamente ao auxílio de um profissional farmacêutico e da equipe multidisciplinar. (FLORES, 2017). Assim, conscientizando a todos os pacientes, sobre uso racional da varfarina, contribui, para a diminuição dos números de interações, intoxicações, interferindo positivamente na adesão e no tratamentopara que seja seguro e eficaz.

Além de questionados sobre quantos medicamentos utilizavam, os mesmos também responderam quais medicamentos eram esses, sendo a maior parte em uso de varfarina que é o mediamento em estudo e o enalapril (90\% e $40 \%$ respectivamente), amiodaona, metoprolol, valsartana, benzetacil, anlodipino, metformina e digoxina sendo utilizados por $10 \%$ dos pesquisados, losartana e sinvastatina (30\%) e aldactone e carvediol (50\%) .

Quanto às interações dos demais medicamentos utilizados com a varfarina, se destaca alguns efeitos colaterais médios a intensos. A sisnvastatina, por exemplo, aumenta o efeito da varfarina, acontecendo também o inverso, afetando o metabolismo da enzima hepática/intestinal CYP3A4, resultando possivelmente num aumento do INR e no risco de rabdomiólise. A espironolactona, por sua vez, diminui os efeitos da varfarina por algum mecanismo desconhecido. Teoriza-se que a interação resulta do auemnto da concentração de fatores de coagulação como resultado da diurese. A amiodarona aumenta os níveis de varfarina ao diminuir o metabolismo, importante evitar o uso de drogas alternativas. A amiodarona também pode aumentar o nível ou efeito da varfarina, afetando o metabolismo da enzima hepática

CYP2C9/10. Todos devem ser usados com moderação e acompanhamento rigoroso (Drug Interactions Checker Medscape Drug Reference Database.)

Foram questionados a respeito também, das reações adversas quando ingeridos os medicamentos, 60\% informaram que tiveram algum tipo de reação. Dentre as reações foram citados hematomas, sangramento ao tomar anti- inflamatórios, sangramento causado por dupla dosagem de varfarina motivada por esquecimento e aumento do fluxo menstrual. Quanto à origem das prescrições medicamentosas, 60\% vieram de consultas pelo Sistema Único de Saúde (SUS).

Em relação ao uso da varfarina, um dos principais problemas, se não o mais sério é o sangramento, que ocorremmais comumente nos tecidos moles, no trato geniturinário e gatrointestinal, o que está relacionado ao grau de anticoagulação (Beyth, 2001). Deste modo, destaca-se também a importância da suspensão do uso deste medicamento ao realizar alguns procedimentos que contenham alto risco de sangramento (Sousa et al.,2021).

Os anticoagulantes orais são umas das drogas com maior interações medicamentosas, uma vez que, seu uso concomitante se tornou bem comum, principalmente entre idosos, e que levando em consideração a alta taxa de prescrição de medicamentos que possuem alto potencial de interagir com a varfarina, se torna imprescindível o máximo de cuidadopara que sejam evitados esses eventos de reações adversas e interações medicamentosas (Teles et al.,2012) .

\section{Conclusão}

Diante dos resultados apresentados, é possível inferir que a Varfarina como anticoagulante com principal objetivo prevenir quadros de IAM, além de ser usado em diversas patologias e condições relacionadas ao sistema cardiovascular e cerebral, é largamente utilizado e eficaz, estando em utilização entre $90 \%$ dos indivíduos que participaram da pesquisa, se mostrando como principal dentre os medicamentos diversos que os mesmos faziam uso.

Além disso, observou-se que grande parte das pessoas entrevistadas tomam algum tipo de remédio, em um tempode pelo menos 12 meses de uso, porém grande parte não realiza nenhuma atividade física. Com isso, observa-se que mesmotendo a 
periodicidade em consultas se faz necessário realizar algum tipo de atividade para o próprio bem-estar e melhora do condicionamento físico.

Importante reiterar que todos são cientes das suas condições e seguem corretamente as indicações médicas em relação a realização dos exames, com isso a alimentação adequada se faz necessário para aliado aos remédios que normalmente já ingerem como a Varfarina, e enalapril que $40 \%$ das pessoas pesquisadas tomam, além de outras como losartana e sinvastatina.

Visto isso, observa-se que a Varfarina sendo um fármaco que se é utilizado com constância e a muito tempo é extremamente necessário para uma prevenção de infarto agudo do miocárdio, embolia sistêmica, entre outros. Na pesquisa em questão observou-se o medicamento era utilizado devido a Acidentes Vasculares Cerebrais (AVC's) que já tiveram, além de problemas cardíacos. Sendo assim, nota-se que a pesquisa é de suma importância no contexto acadêmico bem como para a comunidade científica pois agrega no conhecimento do assunto, levando assim em sequência a sociedade os devidos resultados.

Os resultados obtidos na pesquisa evidenciam a importância do acompanhamento rigoroso em relação ao uso dos medicamentos mencionados, principalmente quando a interação da varfarina leva a efeitos médios a intensos. Nenhum resultado obtido foi absoluto ou pode ser considerado $100 \%$ assertivo, e também foi um estudo limitado por a maioria dos pacientes serem idosos e muitos não terem condições de responder por sí próprio. Sendo assim, se faz necessária pesquisas mais aprofundadas relacionadas ao tema, em maiores populações e amostras, através de coletas de dados mais abrangentes nas unidades básicas de sáude em geral, para a relalização de artigos ou trabalhos futuros.

\section{Referências}

Almeida, L. S. D., Nascimento, M. M. G., Oliveira, D. R., Oliveira, I. V., Detoni, K. B., Caux, T. R., Santos, A. S. A., Rezende, C. P. \&Mesquita, A. R.(2019). Perfil de utilização de medicamentos potencialmente inadequados entre idosos portadores de doença pulmonar obstrutiva crônica. Revista de Ciências da Saúde Básica e Aplicada, Belo Horizonte. 2(1), 29-42.

Andrade \& Glauber (2016). Determinação da teofilina, clindamicina, varfarina e verapamil por amperometria pulsadaem sistema de análise por injeção em batelada usando o eletrodo de diamante dopado com boro. 2016. 82 p. Dissertação (Mestrado) - Programa de Pós-Graduação em Química, Universidade Federal dos Vales do Jequitinhonha e Mucuri, Diamantina..

Barbosa, L. M., Silva, M. S. A. d., Cavalcanti, A. M. F., Nogueira, L. D. d. M., Francisco, M. A., Santos, M. P. d. M., Lima, C. H. R. d.,Negreiros, J. H. C. N., Monteiro, J. L. G. C., \& Laureano Filho, J. R. (2020). Protocolo de tratamento cirúrgico de pacientes em uso de anticoagulante e antiagregante plaquetário. Research, Society and Development. 9(9), 1-17. https://doi.org/10.33448/rsd-v9i9.7726.

Barbosa, R. A., Mendes, P. M. L. Ferro, S. N. \& Pina, J. C.(2018). Atenção farmacêutica a pacientes em uso de varfarina. Saúde e Ciência em Ação - Revista Acadêmica do Instituto de Ciências da Saúde, Goiás. 4(1), 47-70.

Bastos, J. L. D.\& Duquia, R. P. (2013) Um dos delineamentos mais empregados em epidemiologia: estudo transversal. Notas de epidemiologia e estatística, $17(4), 229-232$

Bauer, K. A. (1993). Coumarin-induced skin necrosis. Archives of Dermatology.129(6), 766-768.

Beyth, R. J. (2001). Hemorrhagic complications of oral anticoagulant therapy. Clinics in Geriatric Medicine.17(1),49-56.

Borlina, L. P., Silva, E. L. C., Ghislandi, C. \&Timi, J. R. R.(2010). Conhecimento sobre anticoagulantes orais e seu manejo por médicos de pronto atendimento. Jornal Vascular Brasileiro, Curitiba. 9(2), 24-28.

Borges \&Jossiane Henrich. (2017). Cuidados de enfermagem à pacientes em uso de varfarina. 22f. Trabalho de Conclusão de Curso (Pós-Graduação em enfermagem em terapia intensiva coronariana e em hemodinâmica) - Departamento de Ciências da Vida, Universidade Regional do Noroeste do Estado do Rio Grande do Sul, Ijuí.

Colet \& Christiane de Fátima.(2016). Uso de varfarina em nível ambulatorial: uma coorte de pacientes do sistema público de saúde.

154f. Tese (Doutorado Acadêmico em Farmácia) - Universidade Federal do Rio Grande do Sul, Porto Alegre.

Colet, C. D. F, Amador, T. A. \& Heineck, I. (2016). Acompanhamento farmacoterapêutico de pacientes em uso de varfarina: uma revisão da literatura . Revista Contexto e Saúde, Rio Grande do Sul.17(32), 134-143.

Costa, C. M. F. N., silveira, M. R., acurcio, F. A., Guerra Junior, A. A., 19 Guibu, I. A., Costa, E. A., Karnikowski, M. G. O., Soeiro, O. M., Leite, S. N., COSTA, E. A., Nascimento, R. C. R. M., Araújo, V. E. \& Álvares, J. (2017). Utilização de medicamento pelos usuários da atenção primária do Sistema Único 
de Saúde. Revista de Saúde Pública,51(2), 1-11. https://doi.org/10.11606/S1518-8787.2017051007144.

Dalpiaz, J. et al.(2017). Qualidade de vida de usuários do sistema público de saúde em uso de varfarina. Revista de Epidemiologia e Controle de Infecção, Porto Alegre, RS.7(3),181-188. https://doi.org/10.17058/reci.v7i3.8930.

Drug Interactions Checker - Medscape Drug Reference Database. Medscape Drugs \& Diseases - Comprehensive peer-reviewed medicalcondition, surgery, and clinical procedure articles with symptoms, diagnosis, staging, treatment, drugs and medications, prognosis, follow- up, and pictures. https://reference.medscape.com/drug-interactionchecker.

Esher, A. \& Coutinho, T.( 2017).Uso racional de medicamentos, farmaceuticalização e usos do metilfenidato. Ciência e Saúde Coletiva, Rio de Janeiro.22(8),2571-2580.

Flores, Luiza de Freitas Lima.( 2017). Educação para o uso de terapia anticoagulante oral com variaria em pacientes internados em hospital universitário terciários: avaliação de conhecimento prévio e variáveis relacionadas. Trabalho de Conclusão de Curso (Graduação em Farmácia) - Universidade Federal do Rio Grande do Sul, Porto Alegre.

Instituto Brasileiro De Geografia e Estatística (IBGE). (2020). População estimada de Vitória da Conquista.

Lima, M. G., Álvares J., Guerra Junior, A.A., Costa, E. A., Guibu, I. A., Soeiro, O. M., Leite, S. N., Karnikowski, M. G. O., Costa, K. S.\& Acurcio, F. A. (2017). Indicadores relacionados ao uso racional de medicamentos e seus fatores associados. Revista de Saúde Pública. 51(2), 1-9. https://doi.org/10.11606/S1518-8787.2017051007137.

Menezes, G. O. D. D., Torres, K. B., Pessoa, C. V., Santos, S. L. F. \&Vasconcelos, L. M. O.(2015). Perfil de utilização da varfarina empacientes atendidos na farmácia básica da secretaria de saúde em um município do Ceará. Boletim Informativo Geum, Piauí.6(1), 52- 61.

Pimenta, M. D. C., Viúdes, M. A. A., Figueiredo, T. P., Costa, J. M. \& Martins, M. A. P. (2015). Perfil de pacientes sob tratamento ambulatorial anticoagulante e nível de compreensão sobre a farmacoterapia: estudo exploratório. Revista Saúde, Juiz de Fora. 11(4), 360 - 370.

Riese, F. G. \& Konarzewski, T. L. (2017). Perfil de usuários de varfarina: uma análise a partir de prescrições. Salao do Conhecimento Unijuí, Ijuí .1-4.

Santana, E. C., Vitorino, F. G. \& Suchara, E. A.(2015). Posso usar a varfarina em conjunto com outros medicamentos?. Revista Panorâmica On-Line, Barra do Garças-MT. 18, (1), 36-47.

Soares, A. N., Brandão, E. C., Cunha, G. F., Scherrer, L. R., Precivale, M., Strassmann, P. G. \& Assis, S. R. L. (2011). Estudos sobre Causalidade e Etiologia. In: Moreira, Wagner Brant. (org.). Leitura Crítica de Artigos Científicos. Gramado. 93-102.

Souza, Maria Fernanda da Silva. (2019). Interferência de alimentos ricos em vitamina K na terapia com varfarina em pacientes do Hospital Universitário Onofre Lopes. 39f. Trabalho de Conclusão de Curso (Graduação em Farmácia) - Departamento de Farmácia, Universidade Federal do Rio Grande do Norte, Natal.

Sousa, M. M. C., Bezerra, I. N., Nogueira, F. D., Veras, L. M. C., \& Bezerra, D. M. (2021). O manejo pós operatório do implante do valvar aórtico percutâneo com uso de anticoagulantes e antiagregantes plaquetários: Uma revisão de literatura. Research, Society and Development. 10(6), 1-6. https://doi.org/10.33448/rsd-v10i6.15631.

Souza, T. F. D. (2016). Uso de varfarina: Nível de informação e adesão ao tratamento em pacientes da atenção primária à saúde-Ufrgs. Lume repositório digital. 57f. Dissertação submetida ao Programa De PósGraduação Em Assistência Farmacêutica da Universidade Federal do Rio Grande do Sul.

Souza, T. F. d., Colet, C. F., \& Heineck, I. (2018). Nível de informação e adesão à terapia de anticoagulação oral com varfarina em pacientes acompanhados em ambulatório de atenção primária à saúde. Jornal Vascular Brasileiro. 17(2),109-116. https://doi.org/10.1590/1677-5449.012017 .

Teles, J. S., Fukuda, E. Y. \& Feder, D.( 2012). Varfarina: perfil farmacológico e interações medicamentosas com antidepressivos. Revendo Ciências Básicas, Santo André-SP.10(1),110-115.

World Health Organization (WHO). (2002). The Importance of Pharmacovigilance: safety monitoring of medicinal products. WHO, 48. 